\title{
Nasopharyngeal Foreign Body Following Heimlich Manuever - A Rare Case Report
}

\author{
Authors \\ Shanmugam V.U1, Thulasi Doss ${ }^{2}$, Ruta Shanmugam ${ }^{3}$, Bharath ${ }^{4}$, Prem Nivas ${ }^{5}$ \\ ${ }^{1,3}$ Professor, Department of ENT, Rajah Muthiah Medical College \& Hospital, Annamalai University \\ ${ }^{4}$ Senior Resident, Department of ENT, Rajah Muthiah Medical College \& Hospital, Annamalai University \\ ${ }^{2}$ Postgraduate, Department of ENT, Rajah Muthiah Medical College \& Hospital, Annamalai University \\ ${ }^{5}$ Lecturer, Department of ENT, Rajah Muthiah Medical College \& Hospital, Annamalai University
}

\begin{abstract}
Foreign body inside the nasal cavity is one of the most common otolaryngology emergencies among pediatric population $^{[1]}$. The chances of it getting impacted and retained in the nasopharynx are possible but not frequently encountered. Nevertheless, the nasopharyngeal region should always be looked for impaction of forgotten foreign objects. This report presents an interesting case of a child, who had ingested a foreign body which was dislodged and retained in the nasopharyngeal region following Heimlich Maneuver.

Keywords: nasopharynx, impacted foreign body, metal clip, Heimlich maneuver.
\end{abstract}

\section{Introduction}

Paediatric Aerodigestive Tract Foreign Bodies are a commonly encountered emergencies ${ }^{[2]}$. The nasopharynx or the post-nasal space is the clinically inaccessible space behind the nasal cavity and above the uvulopalatal region ${ }^{[3]}$. However, the nasopharynx is an important site to check and not to miss. Impaction of foreign body in the post-nasal space after being ingested is a rare event. The significance of this region is that it has a less sensitive mucosa lining it and the foreign body may be retained without any signs for a long duration ${ }^{[3]}$. Moreover if the size and shape of the foreign body is such that it does not fall down into the oropharynx and laryngopharynx, it is lodged precisely at this site. The common route of entry is via mouth i.e, the oral cavity and the oropharynx.

\section{Case Report}

A two and half year girl was brought to casualty with complaints of foul smelling nasal discharge from both nostrils for the past seven days. Her mother revealed history of foreign body ingestion one and half years back following which Heimlich maneuver was performed in a private clinic. The child was relieved of her symptoms following the procedure and further evaluation of the upper aerodigestive tract showed no evidence of foreign body and so patient was reassured. This was followed by recurrent upper respiratory tract infection accompanied by foul smelling nasal discharge after one week. However, although this was relieved on medications for the time being, the nasal discharge continued on with attacks of upper respiratory tract infection. 
On general examination, the child was playful, afebrile, with no apparent signs of respiratory distress. On anterior rhinoscopy nasal septum was in midline with foul smelling nasal discharge in both nasal cavities. Examination of oropharynx reveals bilateral grade one tonsillar enlargement. Examination of oral cavity and ear appears normal.

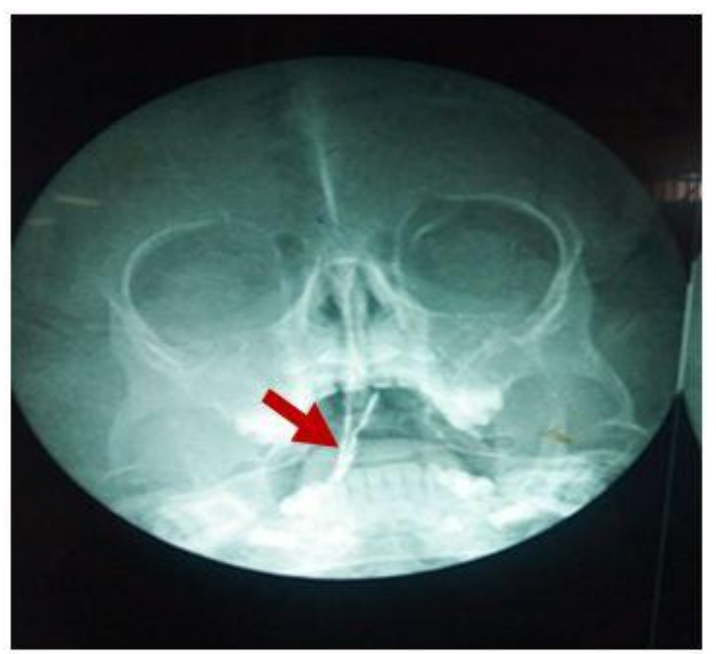

Fig.1 X-ray PNS water's view showing radio opaque foreign inside the mouth/ hard palate region.

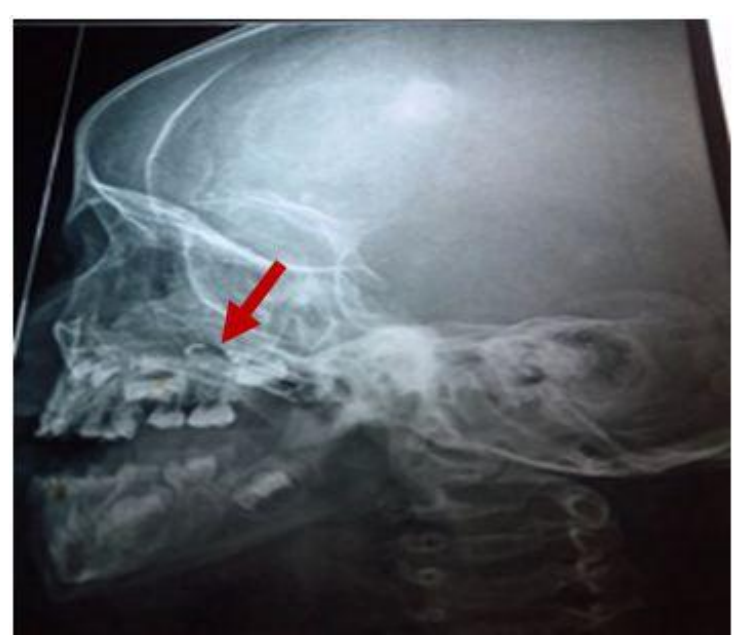

Fig.2 X-ray nasopharynx showing radio opaque foreign body along the floor of nasal cavity and nasopharynx.

$\mathrm{X}$-ray skull lateral view and PNS water's view were taken which showed a radio opaque foreign body visualized in the nasopharynx. Endoscopic examination and removal of foreign body under general anaesthesia was planned for the child.
Under general anesthesia with orotracheal intubation, the patient was placed in Rose's position .A proper size Boyles-Davis mouth gag was introduced and mouth opened, fixed with Draffin's bipod stand. Two rubber suction catheter were used to retract the soft palate to expose the nasopharynx adequately. Using $30^{\circ}$ endoscope the foreign body was visualized in the nasopharynx lodged between the adenoid and posterior end of nasal septum. The foreign body was removed endoscopically using an upturned Blakesley forceps. The postoperative period was uneventful. The patient was discharged after 4 days with oral medications.

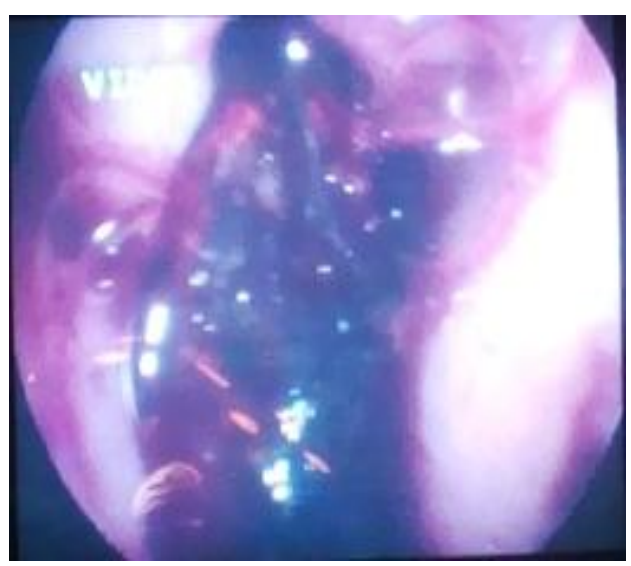

Fig.3 Foreign body visualized impacted on adenoid.

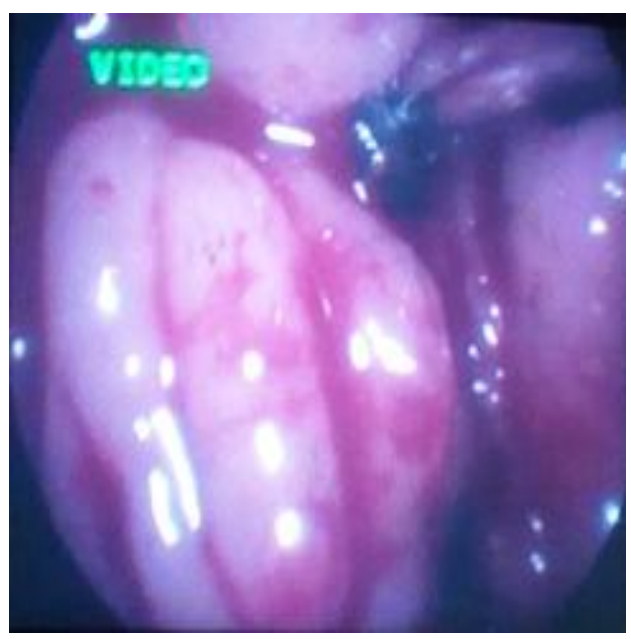

Fig.4 Foreign body being removed by upturned blakesley forceps. 


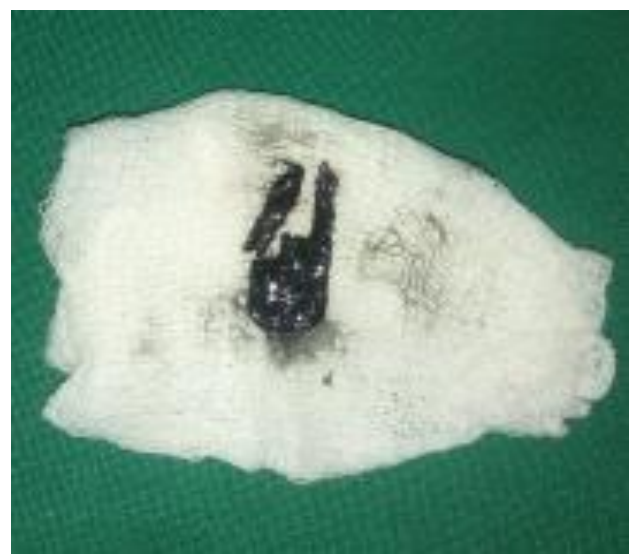

Fig.5 Neglected foreign body after removal

\section{Discussion}

Pediatric age group is notorious for the association with undetected Foreign Bodies in various cavities probably due to Freudian urge. Foreign bodies in the upper aerodigestive tract is classified into Exogenous and Endogenous. Also Traumatic and Non-traumatic, depending on the amount of damage caused by them ${ }^{[4]}$. It is not common for a foreign body to be ingested orally and for it to reach the nasopharynx. An upward ascent of an foreign body into the nasopharynx and its asymptomatic retention for months is a rare presentation. The nasopharynx is an area that is often not looked frequently and thus a potential site for impaction of foreign objects ${ }^{[5]}$. These mostly result from naive attempts at removal by the parents, primary-care physicians, quacks and can occur through regurgitation following vomiting or forceful coughing and that could not be retrieved and with time they are forgotten.

Heimlich maneuver are attempted for aspirated foreign body or food particles. Assuming that there is residual air in the stomach and lungs, a firm two-handed pressure thrust is applied on the epigastrium in the hope that the residual air column created by the thrust will disimpact the foreign body ${ }^{[6]}$. One must remember to clear the mouth and oropharynx manually before application of this maneuver especially dentures.

A non-organic foreign body can remain in the nasopharynx for months especially when there are no symptoms. Foreign bodies in the nasal cavity and post-nasal space may cause unilateral / bilateral purulent nasal discharge, nasal obstruction, chronic rhino sinusitis, cough or may remain asymptomatic ${ }^{[7,8]}$.

If suspected of foreign body in upper airway tract, endoscopic and radiological examination should be promptly performed. A careful history is very informative. Symptoms of foul smelling discharge, nasal obstruction, change in voice, nasal regurgitation with difficulty in swallowing and clinical signs are very important ${ }^{[9,10]}$.

A supportive nasopharyngoscopy should also be a part of investigations as the suspected nasopharyngeal foreign body may not be radio opaque.

In addition to chest and para nasal X-rays, X-ray skull lateral view including nasopharynx is an important radiological investigation for radio opaque foreign bodies. Resuscitation with quick airway management is needed. Trans-nasal removal of foreign body sing endoscopy is safe and effective in most cases ${ }^{[4]}$. Examination of the oral cavity and pharynx including endoscopic examination is recommended to rule out any foreign body remnant and complications like hemorrhage and injury. It is suggested that if a swallowed foreign bodies could not be found anywhere, nasopharynx should be examined.

\section{References}

1. Arijit Jotdar, Mainak Dutta, Subrata Mukhopadhyav - Nasal Foreign Body, Dislodged and Lost- Can The Adenoids Help?-Journal Of Clinical and Diagnostic Research,2015, Jun,Vol-9(6) MD06MD07.

2. Krishnan Ramdoo, Joseph G Manjaly, Taran Tatla- Delayed Presentation of An Undiscovered Nasopharyngeal Foreign Body-et al. BMJ Case Rep 2014.

3. Manish Munjal, Sangeeta, R, S, Greval, Daljit Singh-Paediatric Nasopharyngeal Foreign Bodies- IJO \& HNS, August 1999 (Special Number, F.B).

4. Ravi Kumar, Raju Mudunuri, Durga Prasad Gannavarapu, Achuta Narasimha 
Murty Mukkamala, Jameel K M - Unusual Foreign Body In Nose And Nasopharynx

- A Rare Case Report - Journal Of Clinical And Diagnostic Research, 2015 Apr, Vol-9 (4);MD01-MD02.

5. Arjit Jotkar, Mainak Dutta, Sohag Kundu. Nasopharynx - The Secret Vault For Lost Foreign Bodies of the Upper Aerodigestive Tract - Iranian Journal of Otorhinolaryngology, Vol.28(6), Serial No.89, Nov2016.

6. HJ Heimlich, MA Mason, A W B Crockett, J Coal, E J Trimmer - The Heimlich manoeuvre - British medical journal, volume 28, April 1983.

7. Sunil Kumar, Devendra Bahaur Singh, Abhishek Bahadur Singh, An unusual nasopharyngeal foreign body with unusual presentation as nasal regurgitation and change in voice-BMJ case Rep 2013.

8. Arunabha Sengupta, Pinaki Saha, Subhasish Chakrabarty. Foreign Body In The Nasopharynx of a Child- Indian Journal of Otolaryngology and Head and Neck Surgery, Vol.57, No.3, JulySeptember 2015.

9. A.J.Parker, B.J.Bingham, J.E. OsborneThe Swallowed Foreign Body; Is it in the Nasopharynx?- Postgraduate Medical Journal (1988) 64, 201-203.

10. N.N.Dutta, Bhaben Choudhury -An Unusual Foreign Body in the NaopharynxIndian Journal Of Otolaryngology and Head and Neck Surgery Vol.57, No.3, July-September 2005. 